\title{
Autophagosome formation: not necessarily an inside job
}

\author{
Andrea Longatti ${ }^{1}$, Andrea Orsi ${ }^{1}$, Sharon A Tooze ${ }^{1}$ \\ ${ }^{1}$ London Research Institute, Cancer Research UK, 44 Lincoln's Inn Fields, London WC2A 3PX, United Kingdom \\ Cell Research (2010) 20:1181-1185. doi:10.1038/cr.2010.132; published online 14 September 2010
}

Autophagy is a highly conserved degradative process characterized by the formation of double membrane vesicles called autophagosomes. Autophagy occurs at a basal level in most cells and can be rapidly induced under stress conditions such as amino acid starvation. In multicellular organisms autophagy also plays a role in development, immunity, aging, and tissue homeostasis [1]. Autophagosomes start out as small membrane structures called phagophores or isolation membranes, which are expanded through an unknown mechanism to autophagosomes of various sizes but usually with a diameter of about 300-900 nm. Once mature, autophagosomes fuse with endosomes and lysosomes to form autolysosomes, leading to the degradation of the inner autophagosomal membrane along with all its contents by lysosomal hydrolases. The proteins involved in autophagosome formation were first characterized in yeast, where they are collectively known as Atg proteins. Many of them are conserved in higher eukaryotes. Atg proteins are recruited to, and function on the forming autophagosome sequentially, so that one can follow the formation process over time by following recruitment of the Atg proteins. For example, the Atg5-Atg12-Atg16 complex is recruited to the phagophore

Correspondence: Sharon A Tooze

Tel: +44 207269 3122; Fax: +44 2072693417

E-mail: Sharon.tooze@cancer.org.uk after the serine-threonine kinase Atg 1 (ULK1 in mammalian cells), and the phosphatidylinositol-3 (PI3) kinase complex (Vps34, p150, Atg14 and Beclin1). In turn, the Atg5-Atg12-Atg16 complex is important for the subsequent recruitment of LC3 (Figure 1). LC3, typically tagged with GFP, is often used as a marker for autophagosomes because it remains associated with the autophagosomal membranes until it is degraded in the autolysosome.

One of the most highly debated questions in the field concerns the origin of the autophagosomal membrane [2]. Efforts to answer this question are hampered by the fact that most Atg proteins are only transiently associated with phagophores and autophagosomes. Moreover, it is often difficult to distinguish between the membranes of the autophagosome itself and those of the organelles that have been engulfed to undergo degradation. Many different sources have been suggested since autophagosomes were first described in 1957, including the endoplasmic reticulum (ER) [3, 4], the Golgi apparatus and trans-Golgi network (TGN) [5], and the plasma membrane (PM) [6]. These early studies were descriptive and mostly based on electron microscopic observations.

However, more recent findings have given support to each one of the above hypothesized membrane origins, and outer mitochondrial membranes have been added to the possible candidates [7]. The ER hypothesis has recently gained support by electron tomography studies that showed close association and interconnections between the ER and the phagophore $[8,9]$. Recent studies in yeast cells support the hypothesis that the Golgi and TGN are the origin of the autophagosomal membrane, as Golgi-derived secretory proteins were shown to be essential for autophagosome formation [10-12]. In mammals mAtg9, which is thought to deliver membrane to the phagophore, has been found to localize to TGN and to Rab7, Rab9, and CI-MPR-positive late endosomes [13].

A recent study in the August issue of Nature Cell Biology now provides new evidence for the PM as the origin of the autophagosomal membrane [14]. Ravikumar and colleagues performed a mass spectrometry analysis on cell lysates immunoprecipitated with an antibody against $A \operatorname{tg} 16 \mathrm{~L} 1$, and discovered that both clathrin heavy chain (CHC) and the adaptor protein AP2 co-immunoprecipitate with Atg16L1. $\mathrm{CHC}$ is part of a protein complex that is involved in vesicle budding from the PM, early tubular sorting endosomes, and the Golgi complex. Cargo recruitment to clathrin-coated vesicles on the PM and early endosomes (EE) is mediated by the adaptor protein AP2, while the closely related protein AP1 is specifically found on vesicles budding from the Golgi and the TGN. It would be interesting to know if AP2 is directly mediating the interaction between $\mathrm{CHC}$ and Atg16L, and if so what cargo, if any, 
is recognized by AP2 in the Atg16L1positive regions of the $\mathrm{PM}$.

Furthermore, using siRNA-mediated knockdown of CHC, AP2 and another endocytic protein, Epsin1, they observed a decrease in autophagosomes mostly in basal but also in starvation conditions, thus providing an additional mechanistic link between endocytosis and autophagy. Importantly, siRNA-mediated knockdown of AP1 had no effect on autophagosome formation, excluding an involvement of Golgi-derived vesicles and suggesting a novel and specific role of the clathrin-AP2-Atg16 machinery in autophagy. Moreover, many Atg16L1 positive membranes were found near the PM. All together, these findings prompted the authors to investigate the possibility that the PM itself may provide membrane to autophagosomes.

Cholera toxin B subunit ( $\mathrm{CtxB}$ ) was used to identify endocytic vesicles coming from the PM and to assess whether Atg16L1 was found on these structures. CtxB binds the lipid GM1 on the plasma membrane and is internalized through clathrin-dependent and clathrin-independent mechanisms [15]. CtxB is then transported to the ER, first via EEA1-positive endosomes, and then through the TGN (Figure 1). After 10 min of internalization CtxB co-localizes with Atg16L1 and LC3. However, Atg16L1-positive CtxB vesicles did not co-localize with EEA1, suggesting they are either derived from earlier endocytic carriers or represent later stages of $\mathrm{CtxB}$ transport. Their proximity to the PM would suggest that they are indeed early endocytic carriers. Atg12 and Atg5, which form a complex with Atg16L1, can also be found co-localizing with $\mathrm{CtxB}$, confirming that this is a functional autophagy complex.

Since CtxB is taken up from the extracellular space, it will get trapped in the lumen between the two autophagosomal precursor membranes (Figure 1). Indeed, electron microscopic analysis revealed autophagosomes labeled for both GFP-Atg16L1 and HRP-CtxB. It would be important in the future to understand whether any region of the PM or just GM1-containing CtxB-positive subdomains of the PM can contribute to autophagy. This raises an important issue, which was addressed in the recent papers proposing an involvement of ER [8,9] and mitochondria [7]. In both cases resident proteins from the ER or mitochondria, respectively, were excluded from the newly formed autophagosomes.

To demonstrate that vesicles-containing Atg16L1 are derived from the PM, the authors investigated the effect

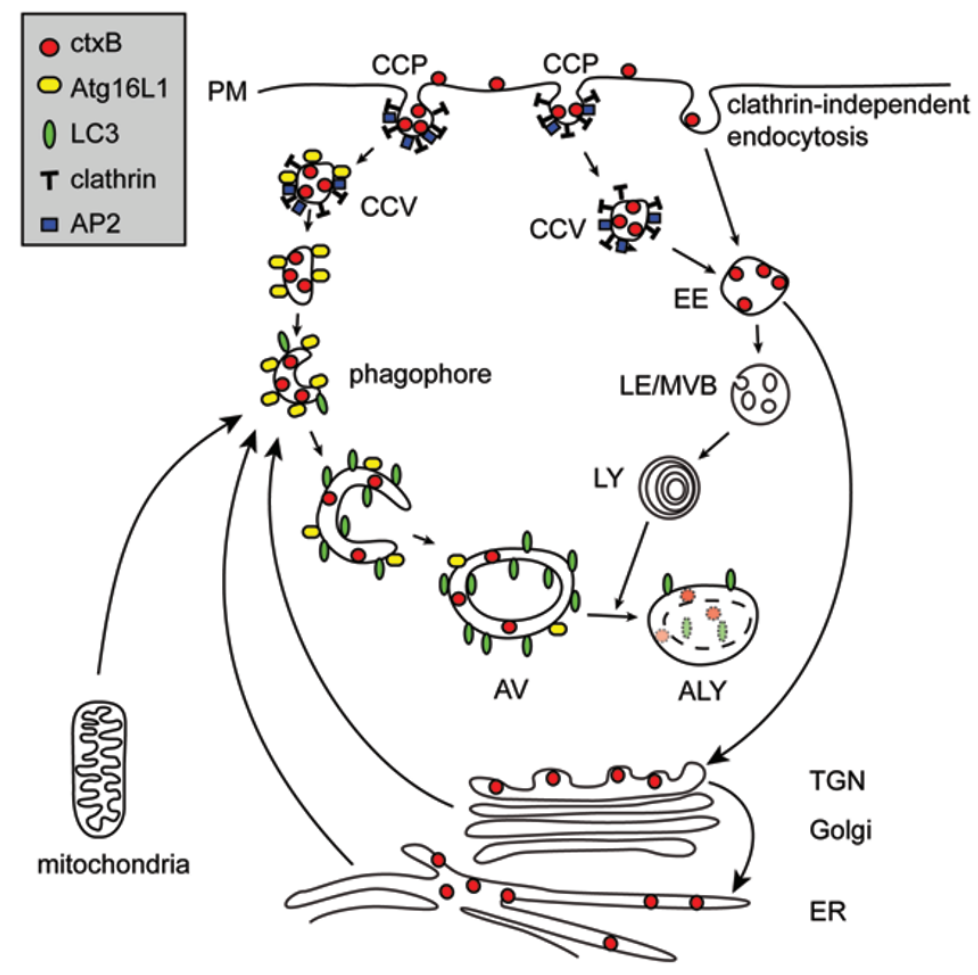

Figure 1 The plasma membrane contributes to phagophore precursor formation. Ravikumar and colleagues provide evidence that endocytic, plasma membrane (PM)-derived clathrin coated vesicles (CCVs) contribute to or form phagophore precursor structures. These are gradually expanded to become autophagosomes, which fuse with endosomes and lysosomes to degrade their contents. Other studies have proposed that the ER, Golgi/TGN, and mitochondria provide autophagosomal membranes supporting the hypothesis that several membrane sources are exploited by autophagy. Knockdown of factors essential for clathrin-mediated endocytosis such as clathrin heavy chain $(\mathrm{CHC}), \mathrm{AP} 2$, and Epsin1 (not depicted) reduces phagophore precursors and autophagosome formation. Cholera toxin B subunit (CtxB) binds to the plasma membrane and is internalized via clathrin coated pits (CCPs) and clathrinindependent mechanisms. CtxB is delivered to the ER via early endosomes (EEs) and the TGN. Using CtxB as a marker for PM-derived endocytic vesicles, they show that these vesicles contribute to the formation of Atg16L1-positive phagophore precursors. Note that in this model, CtxB, and possibly other PMderived proteins as well as extracellular fluids captured in the forming CCVs will be trapped between the inner and outer autophagosomal membrane. The consequence of the presence of this entrapped material, which will eventually be degraded in the autolysosome (ALY), is not known. LE/MVB = late endosome/ multi-vesicular body; $\mathrm{AV}=$ autophagosome. 
of dominant negative (DN) dynamin II and Dynasore, which block Dynamindependent membrane scission. Blocking vesicle scission by these means increased the co-localization of Atg16L1 with clathrin-coated vesicles and the plasma membrane. This suggests that Atg16L1 can be recruited to the PM, where it remains if the vesicles cannot bud off. As Dynamin II has been shown to be involved in vesicle formation from the PM and the TGN, as well as in exocytosis, the precise site-of-action of this DN-protein in this study remains to be directly demonstrated. However, since AP1-dependent pathways have been excluded by the authors, it is unlikely that these findings can be explained by dynamin II activities at the TGN. Similarly, Dynasore inhibits all Dynamins, including the mitochondrial Dynaminlike protein, DrpI and thus experiments using these inhibitors cannot alone differentiate between potentially contributing compartments. Most importantly, in support of the hypothesis put forward by the authors that PM indeed contributes to autophagosome initiation stages, the co-localization between GFP-Atg16L1 and tomato-LC3 was reduced in cells knocked down for Epsin1 or CHC although both populations of puncta are still found. Since the Atg16L1 complex is recruited to phagophores before LC3 and has been shown to determine the location of LC3 lipidation [16], this might suggest that the tomato-LC3positive autophagosomes seen under these conditions form through an Atg16independent pathway.

It would have been of interest to have capitalized on recent developments about the ULK1/2 kinase complex, investigating whether it can be found on these CtxB and Atg16L-positive phagophores or phagophore precursors. It has recently been shown that ULK1 and FIP200 drive the recruitment of all other Atg proteins [17], including Atg14 and the PI3 kinase complex. While inhibition with 3MA (which inhibits PI3P production by the PI3 kinase complex) decreased the number of Atg16L1positive vesicles, the authors did not determine whether PI3P was on the early autophagosomal precursors. This could be resolved by looking at WIPI2, a specific effector of the autophagosomal PI3P pool [18].

An intriguing question that remains is whether these PM-derived vesicles fuse with pre-existing phagophores or whether endocytosed vesicles turn into autophagosomal precursors themselves. The same question would also apply to other donor compartments for autophagosomal membrane. Furthermore, it remains unclear whether PM derived vesicles can contribute to phagophore expansion or only to the formation of the precursor structure. A typical autophagosome of $400 \mathrm{~nm}$ in diameter has a surface area of about $500 \mu \mathrm{m}^{2}$ for each of the outer and inner membranes. In comparison, an average endocytic vesicle has a diameter of less than 100 $\mathrm{nm}$, with a surface area of around 30 $\mu \mathrm{m}^{2}$. This illustrates the quantity of such vesicles needed to form an entire autophagosome. However, given the highly dynamic nature of the PM and endosomal compartments, this may be feasible.

The finding that blocking clathrindependent endocytosis reduces the number of new autophagosomes by $30 \%$ suggests that the PM is not the exclusive source of membrane for autophagy. This is supported by recent publications, and numerous past ones $[3,4,5,6]$. Ravikumar and colleagues [14] provide an intriguing model by which plasma membrane can be recruited to support autophagosome formation. As they suggest, this may be particularly important during enhanced autophagic activity when large amounts of membrane are needed; the PM, being a highly dynamic and large membrane reservoir, seems a reasonable candidate. However, the differential contribution of the PM to autophagy in basal vs. induced conditions was not fully explored and future studies will be required to establish this hypoth- esis. Another possibility is that different cell types use different membranes to form autophagosomes, or that different inducers of autophagy lead to the use of different donor membranes. The recent data certainly support the idea that several pathways for autophagosome formation might exist, including the ER, the Golgi, the mitochondria and the $\mathrm{PM}$, and they could overlap to different extents. Such promiscuity would surely make autophagy an extremely adaptable process, able to function under a wide range of conditions, both ideal properties of an essential survival response.

\section{Acknowledgments}

We thank Cancer Research UK for funding the work in the author's laboratory. We thank the members of the Secretory pathways laboratory for support and advice. We thank Prof Vojo Deretic (University of New Mexico, USA) for his critical reading of the manuscript and discussion.

\section{References}

1 Mizushima N, Levine B, Cuervo AM, Klionsky DJ. Autophagy fights disease through cellular self-digestion. Nature 2008; 451:1069-1075.

2 Longatti A, Tooze SA. Vesicular trafficking and autophagosome formation. Cell Death Differ 2009; 16:956-965.

3 Dunn WA Jr. Studies on the mechanisms of autophagy: Formation of the autophagic vacuole. J Cell Biol 1990; 110:1923-1933.

4 Novikoff AB, Essner E. Cytolysomes and mitochondrial degeneration. J Cell Biol 1962; 15:140-146.

5 Locke M, Sykes AK. The role of the Golgi complex in the isolation and digestion of organelles. Tissue Cell 1975; 7:143-158.

6 Quatacker JR. Formation of autophagic vacuoles during human corpus luteum involution. Z Zellforsch Mikrosk Anat 1971; 122:479-487.

7 Hailey DW, Rambold AS, SatputeKrishnan P, et al. Mitochondria supply membranes for autophagosome biogenesis during starvation. Cell 2010; 141:656-667.

8 Hayashi-Nishino M, Fujita N, Noda T, 
et al. A subdomain of the endoplasmic reticulum forms a cradle for autophagosome formation. Nat Cell Biol 2009; 11:1433-1437.

9 Yla-Anttila P, Vihinen H, Jokitalo E, Eskelinen EL. 3D tomography reveals connections between the phagophore and endoplasmic reticulum. Autophagy 2009; 5:1180-1185.

10 Geng J, Nair U, Yasumura-Yorimitsu K, Klionsky DJ. Post-golgi sec proteins are required for autophagy in Saccharomyces cerevisiae. Mol Biol Cell 2010; 21:2257-2269.

11 van der Vaart A, Griffith J, Reggiori F. Exit from the golgi is required for the expansion of the autophagosomal phagophore in yeast Saccharomyces cere- visiae. Mol Biol Cell 2010; 21:22702284.

12 Yen WL, Shintani T, Nair U, et al. The conserved oligomeric Golgi complex is involved in double-membrane vesicle formation during autophagy. $J$ Cell Biol 2010; 188:101-114.

13 Young ARJ, Chan EYW, Hu XW, et al. Starvation and ULK1-dependent cycling of mammalian Atg9 between the TGN and endosomes. $J$ Cell Sci 2006; 119:3888-3900.

14 Ravikumar B, Moreau K, Jahreiss L, Puri C, Rubinsztein DC. Plasma membrane contributes to the formation of pre-autophagosomal structures. Nat Cell Biol 2010; 12:747-757.

15 Sandvig K, van Deurs B. Transport of protein toxins into cells: pathways used by ricin, cholera toxin and Shiga toxin. FEBS Letters 2002; 529:49-53.

16 Fujita N, Itoh $\mathrm{T}$, Omori $\mathrm{H}$, et al. The Atg16L complex specifies the site of LC3 lipidation for membrane biogenesis in autophagy. Mol Biol Cell 2008; 19:20922100.

17 Itakura E, Noboru Mizushima N. Characterization of autophagosome formation site by a hierarchical analysis of mammalian Atg proteins. Autophagy 2010; 6:764-776.

18 Polson HE, de Lartigue J, Rigden DJ, et al. Mammalian Atg18 (WIPI2) localizes to omegasome-anchored phagophores and positively regulates LC3 lipidation. Autophagy 2010; 6:506-522. 